ISSN: 1130-3743 - e-ISSN: 2386-5660

DOI: http://dx.doi.org/10.14201/teoredu3015374

\title{
ELEMENTOS ONTOLÓGICOS DEL PENSAMIENTO CRÍTICO $^{1}$
}

\author{
Ontological elements of critical thinking
}

\section{Éléments ontologiques de la pensée critique}

\author{
Angélica María RodríGuez OrTiz \\ Universidad Autónoma de Manizales. Facultad de Estudios Sociales y Empresariales. \\ Departamento de Estudios a Distancia. Programa de Maestría en enseñanza de \\ las ciencias. Antigua estación del ferrocarril (Manizales, Colombia). \\ amrodriguez@autonoma.edu.co
}

Fecha de recepción: enero de 2018

Fecha de aceptación: marzo de 2018

\section{RESUMEN}

Este artículo presenta una investigación analítica cuya pretensión consiste en desvelar la naturaleza del pensamiento crítico en aras de aportar claridad conceptual a los discursos que circulan en la literatura sobre ciencias de la educación. Desvelar la ontología ${ }^{2}$ del pensamiento crítico permitirá a docentes e investigadores, en el campo de la educación, adquirir la comprensión necesaria para pensar en estrategias

1. Este artículo hace parte de una investigación mayor, cuyo método analítico permite aclarar algunos conceptos usados en los discursos pedagógicos y didácticos, con el fin de indagar acerca de los procesos de interacción social e interactividad que permiten generar procesos de construcción del conocimiento y desarrollar habilidades de pensamiento crítico. Investigación adscrita al grupo: SEAD-UAM en la línea Educación y Desarrollo.

2. Se entiende por ontología (ontología): el estudio sobre las condiciones de posibilidad de la existencia de lo que le es propio a la naturaleza del ente (la cosa en tanto que existente [ens) o fenómeno (la idea de la cosa). 
pedagógicas y didácticas que jalonen, en los seres humanos, el desarrollo de habilidades de nivel superior, con el fin de formar seres capaces de pensar por sí mismos y de actuar bajo razones y criterios argumentados; discernimientos sustentados en el uso del lenguaje y sus relaciones con el pensamiento y la acción.

Palabras clave: pensamiento crítico; ontología; lenguaje; pensamiento; acción.

\section{SUMMARY}

This article presents an analytical research, in which is to reveal the nature of critical thinking to identify the elements that constitute it and bring clarity to the discourse of Science education literature around this phenomenon. Unveiling the ontology of critical thinking will allow teachers and researchers from different disciplines in the field of education to acquire the understanding of this phenomenon to think didactic and pedagogic methodologies that draw human beings in training the development of higher level skills, in order to reach the formation of autonomous beings, capable of thinking for themselves and acting under argued reasons and criteria, which are based on the use of language and its relations with thought and action.

Key words: Critical Thinking; Ontology; Language; Thought; Action.

\section{SOMMAIRE}

Cet article présente une recherche analytique, qui, à partir d'une étude conceptuelle, vise à révéler la nature de la pensée critique afin d'identifier les éléments qui constituent et apportent clarté aux discours de la littérature des sciences de l'éducation autour de ce phénomène. Dévoiler l'ontologie de la pensée critique va permettre aux enseignants et chercheurs de différentes disciplines du domaine de l'éducation, de comprendre ce phénomène pour appréhender les possibles outils et méthodologies qui permettent, chez les personnes en pleine formation, le développement des compétences de niveau supérieur. Ceci dans le but de former des personnes autonomes, capables de penser par elles-mêmes et d'agir de façon argumentée tout en s'appuyant sur la langue en la mettant en relation avec la pensée et l'action.

Mots clés: pensée critique; ontologie; langage; pensée; l'action.

\section{PANORAMA DEL PENSAMIENTO CRítiCo EN LA EDUCACiÓN}

Gran parte de la reflexión que se ha generado en las dos últimas décadas, en las denominadas ciencias de la educación, ha estado orientada en torno al desarrollo y a la potenciación del pensamiento crítico. Es frecuente auscultar en los discursos sobre enseñanza de las ciencias y pedagogía la discusión acerca de la necesidad de formar pensadores críticos, e iniciar procesos de enseñanza que 
propendan por el desarrollo de habilidades de pensamiento en niveles superiores. En la misma línea se plantean reestructuraciones de diseños curriculares sobre la "urgencia» o "necesidad» de formar a las nuevas generaciones capaces de pensar autónomamente de forma razonada y razonable, lo que conlleva a cambios en las políticas educativas con el fin de promover e implementar estrategias y métodos que enseñen a jóvenes y niños a pensar críticamente.

Ante la situación, Latinoamérica no es la excepción. Países como México, Argentina, Ecuador, Chile, Brasil, entre otros, trabajan en función de transformar los procesos en el aula, a partir de los lineamientos estipulados sobre el pensamiento crítico. En Colombia, por ejemplo, el MEN (2009)3 ${ }^{3}$, en relación al currículo de educación superior, consideró que el pensamiento crítico era una competencia genérica, dadas las necesidades particulares del contexto ${ }^{4}$. Dichas situaciones nos llevan a confirmar lo expuesto, hace unos años, por Antonio Martín y Óscar Barrientos, para quienes: «existe la creencia de que el pensamiento crítico, en su creciente abstracción, tiene aplicaciones recientes en el marco del contexto educativo. En parte eso es así porque se tiene conciencia clara y acotada del término solamente desde un contexto contemporáneo. No obstante, el pensamiento

3. Según el MEN (Ministerio de Educación Nacional) el pensamiento crítico se caracteriza por: Comprensión: contenido y puntos de vista del escritor o del interlocutor. Análisis: identificación de líneas de razonamiento, evidencia, conclusiones, argumentos. Identificación de deficiencias en argumentos: inconsistencias lógicas, supuestos infundados, consecuencias no intencionadas, recursos retóricos distorsionantes, falsas analogías, etcétera. Evaluación: credibilidad y validez de la evidencia, credibilidad de líneas de razonamiento, validez de argumentos, solidez de las conclusiones, etcétera. (MEN, 2009, 10).

4. La situación no es ajena a otros países de Latinoamérica. Para el caso de Ecuador se puede evidenciar una situación similar, puesto que el Ministerio de Educación Ecuador realizó en 2009 un Curso de Didáctica del Pensamiento crítico, el cual, en 2011, fue repensado y ajustado de acuerdo con las necesidades contextuales, para trabajarlo en el Programa de formación continua del Magisterio Fiscal. En Chile, por ejemplo, el programa BETA-PUCV (2005), expone que busca desafiar a los alumnos a potenciar sus capacidades cognitivas y a mantener motivación por el aprendizaje, con el fin de romper los paradigmas regulares de los sistemas de educación, y encaminar a estos alumnos dotados hacia un pensamiento autónomo, propio, crítico. El programa se plantea desde los lineamientos de FACIONE (1990) y PORTO (2000), entre otros autores, para quienes el currículo no debe ser concebido como parcelas aisladas, sino que debe integrar el desarrollo de todos los conocimientos y destrezas de nivel superior. En otras palabras, las habilidades y las destrezas de nivel superior son el objetivo en los procesos de formación. Razón por la cual, un elemento fundamental en este programa es la vinculación de la filosofía, ya que esta disciplina propende por un pensar crítico y brinda herramientas desde la lógica para estructurar mejor el pensamiento. En México también puede revisarse el trabajo realizado por diferentes instituciones en torno al desarrollo de habilidades de niveles superiores. Los trabajos realizados en el CECAPFI, en la Universidad de Morelos y en la Universidad Autónoma Nacional de México, son una muestra de ello. Trabajos que asumen posturas críticas como el de López (2012), quien, además de exponer la importancia de formar en pensamiento crítico, plantea algunas dificultades de su uso en los contextos escolares. En la misma ruta de trabajo, The International Commission on Education for the 21st Century ha presentado diversos informes a la UNESCO (1996) en los que expone que «el desarrollo del pensamiento crítico de los alumnos es esencial para favorecer una verdadera comprensión de los acontecimientos entre los alumnos, en lugar de desarrollar y mantener una visión simplificadora de la información relacionada con estos acontecimientos» (FRANCE et al., 2003, 532). 
crítico ha sido siempre un paradigma presente en el campo educacional (quizá bajo otros nombres o sugerido por otros soportes teóricos)» (Martín y Barrientos, 2009, 21).

En la misma dirección, Nickerson (1994) y Shannon y Allen (2001) exhiben la preocupación latente de investigadores y educadores del siglo xxI, que gira en torno al desarrollo de habilidades de pensamiento de orden superior de los estudiantes. Habilidades necesarias para dar soluciones a los problemas sociales y escolares propios del entorno en el que se desenvuelven los estudiantes. Pese a dichas reflexiones y discursos, las realidades sociales, políticas y económicas que se presentan en las democracias pluralistas explicitan en las acciones humanas gran falta de las mismas; conviene revisar las estadísticas sobre las masivas abstenciones de participación ciudadana en las jornadas electorales de los últimos años, así como el desconocimiento de los mecanismos de participación ciudadana, o la toma de decisiones en situaciones políticas, para confirmar que urge la formación de seres críticos, autónomos, capaces de tomar postura y asumir responsablemente su papel como ciudadanos del mundo. Tal vez por ello, ahora más que antes, «el pensamiento crítico se ha tornado una tradición de investigación y de enseñanza de origen filosófico y de desarrollo multidisciplinario" (Martín y Barrientos, 2009, 22), lo que hace evidente un requerimiento esencial en el campo de la educación y en sus diferentes niveles. Como bien lo cuestiona Facione (2007): "¿No es una política social más sabia invertir en la educación de la fuerza laboral del futuro, que sufrir las pérdidas financieras y soportar las cargas fiscales y sociales relacionadas con la fragilidad económica, los problemas de salud pública, el crimen, y la pobreza evitable?» (p. 1). Parece, por todo lo dicho anteriormente, que será mejor educar para pensar críticamente, antes que pasar indolentes ante la realidad social que nos aqueja.

Ahora bien, en medio del florecimiento de discursos en torno al tema, autores como Facione (1990 y 2007), Olivares, Saiz y Rivas (2005), Montoya (2007), Wacquant (2006), Paul y Elder (2003), López (2012), Martín y Barrientos (2009), Zoller (1991), Nickerson (1994), Shannon y Allen (2001), entre otros, constituyen el sustento teórico de los informes de investigaciones que circulan en las revistas especializadas en torno a las prácticas de aula en las que se exponen planteamientos, argumentos y reflexiones en torno a las características de un pensador crítico, dimensiones de este tipo del pensar bumano, babilidades que intervienen en su desarrollo, formación de pensadores críticos, tipos de razonamientos y procesos mentales que se promueven, evaluación del pensamiento, entre otros temas. Sin embargo, en la divulgación literaria en torno al pensamiento crítico no hay un estudio que desvele la naturaleza del pensamiento crítico, es decir, que estudie su ontología. O dicho de otro modo: aun cuando este se acuna en la filosofía misma y se realicen recorridos históricos que así lo desvelan, no se ha realizado el tipo de estudio sobre la esencia de lo que es el pensamiento crítico. Cabe resaltar que gran parte de los autores mencionados convocan estudios desde la génesis de este 
como proceso esencial en la formación humana ${ }^{5}$ en la tradición filosófica; como es el caso de Facione (1990 y 2007) y Martín y Barrientos (2009), quienes realizan y demarcan rastreos de las teorías filosóficas, en especial de los pensadores de tradición racionalista para dar sustento a los orígenes del mismo.

Visto así, y ante el discernimiento en torno al tema desde las reflexiones expuestas en las ciencias de la educación, es preciso aseverar que los estudios que competen a la filosofía en torno al pensamiento crítico y la formación serán siempre de orden ontológico, teleológico y epistemológico, cada uno de ellos desde una mirada analítica, con el fin de clarificar el concepto y con ello el discurso, así como aportar en la comprensión del fenómeno mismo. Pese a ello, es preciso decir que ha sido la didáctica quien en los últimos años ha planteado una reflexión teleológica y epistémica no solo desde la didáctica en general, sino desde los nuevos planteamientos de las didácticas específicas, en las cuales, día a día el pensamiento crítico cobra una significativa relevancia en los procesos de transformación de la enseñanza y el aprendizaje en dominios específicos.

En vista de lo anterior, una vez demarcado el camino de la discusión y tras reconocer el aporte que han realizado en este estudio disciplinas como la psicología, las neurociencias, la pedagogía y las didácticas, es hora de que desde la filosofía se empiecen a delimitar los fundamentos ontológicos del fenómeno, es decir, se tienda a volver sobre la naturaleza del pensamiento crítico, para analizarlo en sí mismo e iniciar una reflexión conceptual en torno a esta forma específica del pensar humano, que demanda el desarrollo de habilidades de nivel superior.

Antes de iniciar el análisis conceptual para comprender lo que es el pensamiento crítico en su naturaleza, cabría la cuestión siguiente: ¿Cuál sería el aporte de este estudio para las ciencias de la educación y para el resto de disciplinas que reflexionan en torno al tema? Pues bien, ante dicha cuestión, es preciso aclarar que realizar un estudio ontológico del pensamiento crítico aporta a la discusión en la enseñanza de las ciencias, en la pedagogía y en la educación, en general, en la medida en que le brinda al docente mayor claridad conceptual para referirse al

5. Gran parte de la reproducción del discurso sobre el pensamiento crítico expone conceptualmente, desde un anclaje histórico, la importancia de este; para ello, perpetra la génesis en la filosofía. Y es que la historia de la pedagogía y la filosofía de la educación nos remite al ideal de la Paideia griega, el cual pretendía formar pensadores críticos. En el rastreo histórico, se desvela que uno de los grandes exponentes de estos referentes iniciales sobre el pensamiento crítico es Platón, quien en su academia formaba pensadores libres, usando como estrategias la mayéutica, en la que el diálogo y la discusión movían a los sujetos a la reminiscencia, generando espacios para el reconocimiento de la verdad que se alcanzaría a través del alma. La República de Platón y cada uno de los diálogos menores permiten interpretar cómo en el ideal griego se tendía a la formación de espíritus libres; en esa misma línea se pueden referenciar una serie de autores destacados que en sus escritos brindan herramientas conceptuales y metodológicas sobre la formación de pensadores críticos; entre ellos, en la Ilustración pueden reconocerse los planteamientos kantianos sobre la autonomía y la deseada mayoría de edad, elementos que aún permanecen vigentes en las disertaciones sobre el tema. Es innegable que la filosofía misma en su naturaleza apueste por pensadores capaces de pensar por sí mismos, de asumir posturas críticas y de plantear posibles soluciones a las cuestiones más esenciales de la humanidad. 
fenómeno mismo con el fin de mejorar procesos de enseñanza y aprendizaje. La comprensión de la naturaleza le brindará a docentes e investigadores de aula herramientas conceptuales y metodológicas para crear estrategias que jalonen el desarrollo de ciertos tipos de habilidades de nivel superior del pensamiento humano, y se intuya la naturaleza de las formas de pensar necesarias para que los seres humanos alcancen el espíritu crítico que el mundo de las nuevas tecnologías, los sistemas de la información y las democracias pluralistas demanda. Por otra parte, un estudio ontológico del pensamiento crítico permite a investigadores y educadores vislumbrar este fenómeno que se ha puesto de moda en los discursos de la educación, con el fin de que, por sí mismos, empiecen a examinar mejor las posturas que lo sustentan y se construyan discursos con sentidos y significados críticos, ya que el término es usado sin mayor conciencia sobre lo que este significa y convoca.

\section{NATURALEZA DEL PENSAMIENTO CRÍTICO}

Como se ha enunciado hasta el momento, en la mayoría de trabajos e investigaciones en torno al pensamiento crítico se da por hecho la existencia de este fenómeno; existencia que se sustenta en teorías de corte racionalista, o bien platónicas, en las que se expone como un ente independiente, cual producto acabado -dado que, para algunos teóricos, el pensamiento crítico se alcanza como se alcanza la verdad o el conocimiento, es decir, a través de la razón, a través del alma $-{ }^{6}$ o bien desde posturas innatistas enmarcadas en la teoría de Descartes, en las que el pensamiento crítico se concibe como una cualidad o disposición del alma, una disposición innata al ser humano -una forma de razonar propia a la naturaleza humana- o desde planteamientos kantianos, postura que tal vez demarca un justo medio entre las dos anteriores, pues expone el desarrollo del pensamiento crítico en el ejercicio permanente de la razón, planteamientos ontogenéticos que se quedan más en lo segundo que en lo primero, es decir, que centran su atención en la génesis más que en lo óntico.

En la literatura especializada se puede inferir que los estudios sobre la génesis del pensamiento crítico en los procesos formativos nos remontan, como lo han mostrado Tamayo (2007) y Martín y Barrientos (2009), a revisiones historiográficas en la filosofía. Es indudable que el pensamiento crítico es una condición necesaria para hacer filosofía. La crítica siempre ha estado y estará presente en la naturaleza de la reflexión filosófica, pues todo aquel que se dedique a filosofar ha de ser, por naturaleza, un ser crítico si pretende emprender un camino serio en la búsqueda del conocimiento. Conviene revisar las habilidades que se han postulado como esenciales a la hora de formar en pensamiento crítico, habilidades que fueron

6. En algunos textos se presenta el pensamiento crítico como una entidad deshumanizada, como si existiera aparte del sujeto humano, y en los procesos de ejercitación de la razón se alcanzase. No se ve como un proceso mental, elaborado por seres humanos. 
expuestas en la taxonomía de Bloom et al. (1956), para ver que el tema no es algo nuevo. Las mismas habilidades de nivel superior que el autor identifica están presentes en el ejercicio filosófico desde su origen -en el paso del mito al logos- dichas habilidades son condiciones sine qua non de la filosofía. Tal vez por ello, parte de las investigaciones realizadas sobre habilidades para pensar críticamente en el aula centran su atención en elementos propios de la gnoseología y de la epistemología; los cuales, han sido ejes fundamentales en la construcción del conocimiento y el trasegar científico ${ }^{7}$. Lo único que se ha hecho, como se esboza en la literatura cultivada, es un giro de acomodación de los lineamientos gnoseológicos a los pedagógicos y didácticos, según las necesidades y los discursos.

La cimientos de las teorías sobre procesos de nivel superior en la tradición filosófica nos remontan a ciertos elementos -la dialéctica, la indagación, la ironía- de la mayéutica socrática, así como a los silogismos de la lógica formal y los planteamientos de la ética aristotélica. En ese mismo orden, también es posible identificar elementos que sustentan la crítica desde la teoría racionalista cartesiana expuesta en sus Meditaciones acerca de la Filosofía Primera (Descartes, 2014) y, por supuesto, los postulados del pensamiento crítico, de la autonomía y la responsabilidad en la obra de $\mathrm{Kant}^{8}$ (2011); asimismo, hay elementos de la dialéctica hegeliana (Hegel, 2010) y marxista (Marx, 2014), así como ideas de la filosofía del lenguaje; componentes que se constituyen en fundamentos esenciales a la hora de iniciar procesos de ejercitación para pensar críticamente. Podría decirse que en estos autores y corrientes se sustentan estrategias y metodologías actuales que abogan por formar ciudadanos capaces de pensar por sí mismos y plantear posibles soluciones a los problemas que les aquejan en la realidad que habitan. En otras palabras, «es misión de la educación poner las emociones nobles e irracionales del alma en tal pie de armonía con la parte espiritual del hombre que, apoyándose en ella, lo verdaderamente humano, a pesar de ser tan débil, sea capaz de tener a raya lo infrahumano» (Jaeger, 1980, 760). De ahí la importancia de que se formen seres críticos, autónomos, capaces de actuar racionalmente, y orientar sus acciones individuales hacia al bien social y de que la filosofía esté presente a la hora de

7. TAmayo et al. (2011), por ejemplo, proponen un modelo de unidades didácticas para la enseñanza de las ciencias en cada campo del saber. Modelo en el cual el elemento histórico epistemológico está presente no solo en la investigación, sino en la transposición didáctica. Cada docente investigador debe rastrear el desarrollo de las habilidades del pensamiento crítico a lo largo del proceso y en cada fase. "Al ser la enseñanza una actividad que involucra distintas entidades y no una actividad de transmisión de información, vemos la necesidad de abordar la educación de las ciencias desde una perspectiva constructivista y evolutiva, en la cual se integren aspectos tales como: la historia y epistemología de los conceptos, las ideas previas de los estudiantes, la reflexión metacognitiva, los múltiples lenguajes que incluyen las TIC y el proceso de evolución conceptual como aspecto que permite una evaluación formativa, la transformación del conocimiento del pensamiento inicial y final de los docentes y de los estudiantes" (p. 106).

8. Tal vez los postulados de la crítica kantiana son los más usados en la diversa literatura cuando se habla de pensamiento crítico; sin embargo, es preciso reconocer que no es el único autor que ha trabajado en este campo, y que su teoría tiene bases en la filosofía clásica que tuvo origen en Grecia. 
analizar, aplicar, comprender, conocer, sintetizar y evaluar las situaciones a las que se enfrenta (Bloom et al., 1956).

Para dejar de lado la génesis de la reflexión sobre el pensar crítico e introducirnos en el estudio óntico se nos demanda un análisis conceptual, cuya pretensión se enfoca en la comprensión de los significados otorgados al pensamiento crítico, siendo este -el análisis- la primera forma de indagar por el fenómeno, puesto que nos lleva a pensar la naturaleza desde lo que este significa.

El pensamiento crítico, en primera instancia, implica, como lo expuso Facione (2007), cuestionar si se debe entender "como un buen juicio, casi lo opuesto a pensamiento ilógico, irracional»; esta definición acaba por ser insatisfactoria; ya que, si bien el juicio9 racional es un elemento central del pensar bien, y críticamente, no es suficiente por sí mismo para definir lo que es el pensamiento crítico ni determinar qué subyace a su esencia. Yendo más allá, podríamos hacer alusión a la definición a la que llegaron grandes pensadores y que está expuesta en el informe APA Delphi, definición alcanzada por consenso de los expertos, dentro de la cual se considera como «el proceso del juicio intencional, autorregulado. Este proceso da una consideración razonada a la evidencia, el contexto, las conceptualizaciones, los métodos y los criterios». Definición sustentada en los planteamientos de Kant, para quien «la crítica de la razón conduce pues, en último término, necesariamente a la ciencia; en cambio, el uso del dogmático de ella sin crítica [conduce] a afirmaciones sin fundamento, a las que pueden oponérseles [otras] igualmente verosímiles, y, por tanto, conduce al escepticismo» (Kant, 2001 [Krl], B23). Puesto que, además de ser mayormente amplia, nos permite empezar a pensarlo como un proceso mental de orden superior, que involucra, además de la razón, métodos y conocimiento para definir los criterios en la toma de postura. Así, la noción de pensamiento crítico nos lleva a transitar entre la razón, la intencionalidad y la conciencia, con el fin de alcanzar la regulación; asimismo, nos permite indagarnos por el conocimiento, pues los juicios se formulan con base en los conocimientos sobre el objeto determinado, así como las evidencias y los criterios; a esta definición se le suma el método, el cual, al parecer, es de carácter lógico, con el fin de alcanzar los mejores juicios y conceptualizaciones al asumir y defender una postura crítica y propia.

Por otra parte, para Paul et al. (2005), el pensamiento crítico se entiende como el proceso intelectualmente disciplinado de activar y hábilmente conceptualizar, aplicar, analizar, sintetizar y/o evaluar información recopilada o generada por

9. Tal y como se ha expuesto, gran parte de los referentes teóricos en torno al pensamiento crítico se fundamentan en las posturas kantianas. "Juicio" es un concepto fundamental de la teoría de la Crítica de la razón pura de Kant, pero este por sí solo es insuficiente si no se sustenta en la razón. "La misma función que da unidad a las distintas representaciones en un juicio proporciona también a la mera síntesis de diferentes representaciones en una intuición una unidad que, en términos generales, se llama concepto puro del entendimiento» (KANT, 2011 [KrV], A79/ B105). En otras palabras, sin razón no sería posible hablar de lenguaje, no sería posible hablar de lógica y mucho menos sería posible hablar de conocimiento ni de ciencia. En la teoría kantiana el eje que regula el pensamiento a través de la lógica es la razón. 
observación, experiencia, reflexión, razonamiento o comunicación, como una guía hacia, la creencia y la acción definición que involucra dos elementos más de los anteriormente expuestos: creencia y acción. Este, además de ser un planteamiento cercano a la postura wittgensteiniana ${ }^{10}$ al tomar razón y comunicación como iguales. Para Wittgenstein pensamiento y lenguaje son lo mismo; el pensamiento está dado en términos lógicos, en términos de racionalidad. "El pensamiento contiene la posibilidad del estado de las cosas que piensa. Lo que es pensable es también posible [...] No podemos pensar nada ilógico, porque de lo contrario tendríamos que pensar ilógicamente» (Wittgenstein, 2009, af: 2.221; 2.22), toma el razonamiento y la comunicación como lo mismo. Asimismo, delinea en cada proceso al que hace referencia al lenguaje, puesto que estos procesos de pensamiento que son reconocidos en las ciencias cognitivas como procesos de nivel superior solo son posibles en el uso del lenguaje.

En el mismo sentido, exponen autores como Beltrán y Torres (2009) que el pensamiento crítico, como proceso cognitivo, permite la construcción de un nuevo conocimiento y la utilización estratégica del mismo en la solución de problemas presentes en la vida cotidiana. Facione (2007) afirma que el pensamiento crítico se relaciona con la razón, la honestidad y amplitud mental, elementos que son llamados por como características intelectuales del pensador crítico. Es decir que, en su mayoría, los autores expuestos y que han sido tradicionales en la literatura con relación a este tema exponen el pensamiento crítico como un proceso de pensamiento de orden superior, en el cual el agente posee ciertas características esenciales que le permite resolver problemas, asumir posturas críticas, analizar, sintetizar, reflexionar, entre otras, con el fin de plantear posturas argumentadas para actuar de manera razonada y razonable.

Ahora bien, bajo esta concepción que toma al fenómeno del pensar crítico como proceso hay ventajas y desventajas. Algunas ventajas se plantean en la medida en que en todo proceso hay movimientos, cambios y transformaciones, es decir, que no es acabado; por lo cual, estamos en una movilización de las habilidades de pensamiento, que debe ser permanente, en un ejercicio constante y en el que nos comprometemos como sujetos críticos. Asimismo, hay desventajas, ante todo para las posturas positivistas que circundan en las ciencias cognitivas experimentales, que pretenden medir los niveles críticos alcanzados, ya que un proceso es un movimiento hacia adelante, en evolución cambiante de acuerdo a las exigencias del medio; por lo cual, nunca alcanzaríamos a determinar el nivel de pensamiento crítico que un ser humano puede alcanzar, ni el nivel de desarrollo que se requiere en las habilidades del pensar crítico, ello sin contar con la postura innatista que considera que dicho proceso es propio de la naturaleza racional del ser humano, en cuyo caso no habría movimiento alguno, ni se alcanzarían niveles, puesto que ya está determinado su alcance de acuerdo con la naturaleza humana.

10. En el Tractatus lógico-philosophicus se puede ver que para Wittgenstein lenguaje y pensamiento son lo mismo: «El pensamiento es la proposición con sentido» (WiTTGENSTEIN, 2009, af: 3.325). 


\section{ELEMENTOS DEL PENSAMIENTO CRÍTICO}

Una vez analizadas algunas de las definiciones más representativas ${ }^{11}$ en la literatura sobre el pensamiento crítico e identificar algunos conceptos centrales que sostienen teóricamente las mismas, se puede delimitar el estudio ontológico. La revisión conceptual permite identificar algunos términos comunes en el proceso del pensar críticamente, entre ellos: juicio, intelecto, razón, intencionalidad, conciencia, creencias, lenguaje, conocimientos y acción. Sería muy arriesgado decir que las tres primeras, al igual que las tres siguientes, forman parte de la mente humana, dado que las tres primeras podrían tal vez enmarcarse en el entendimiento humano como facultades esenciales del pensamiento, mientras que las tres siguientes (intencionalidad, conciencia y creencias) pertenecen a la mente, a lo que se ha denominado en la filosofía de la mente estados mentales; asimismo, podría decirse que el lenguaje y el conocimiento acaban por ser transversales a la mente y al pensamiento, ya que el contenido del pensamiento y de los estados mentales está dado en términos lingüísticos y representacionales. Las representaciones están en relación directa con el conocimiento que el agente posee ${ }^{12}$ y este es expresado en términos proposicionales. Un último elemento sería la acción, en la cual se evidencian los elementos anteriores; por lo cual, podría decirse que ante lo descrito hay tres elementos esenciales en la naturaleza del pensamiento crítico, y estos tres abarcan los demás: lenguaje, pensamiento y acción.

La categorización no es arbitraria. Se sugiere poner en primera instancia el lenguaje y es una decisión intencional, puesto que como elemento biosocial está en la naturaleza del pensamiento mismo. No hay pensamiento sin lenguaje, los juicios, la racionalidad, el intelecto está dado en términos del lenguaje. Para el caso de los estados mentales: intencionalidad, conciencia y creencias el lenguaje hace parte de la propia naturaleza de la mente, tal y como lo expone Rodríguez (2016) ${ }^{13}$ la mente humana es de naturaleza biopragmática ${ }^{14}$. Puesto que los estados mentales

11. Es preciso aclarar que se toman como las más representativas, dado que las investigaciones, congresos y discursos en torno al pensamiento crítico siempre se fundamentan en las definiciones de estos autores. Se reconoce que hay algunas otras más, pero todas están en relación con las anteriormente descritas.

12. Toda creencia está dada en términos lingüísticos. Las creencias como estados mentales tienen un contenido epistémico, puesto que son las que permiten comprender los fenómenos, las situaciones, el mundo para actuar en él.

13. Hablar de una explicación biológico-pragmatista de la conciencia implica reconocer la emergencia natural de la conciencia en su más bajo nivel, así como la relación entre lenguaje, racionalidad y acción en los niveles superiores, y afirmar la subjetividad de la misma, sin llegar a reducir la conciencia ni al cerebro, ni a la conducta, ni al lenguaje; se plantea entonces que la conciencia en sí misma no es estática e inmutable; sino que por el contrario está en permanente movimiento y desarrollo para alcanzar los grados más altos y complejos como estado mental (RODRíGuEz, 2016, 232).

14. Biopragmático: hace referencia a aquellos fenómenos, hechos o acontecimientos que se presentan en el mundo, cuya naturaleza está sustentada en la biología, la pragmática del lenguaje y las vivencias sociales (expresada en las acciones); es decir, para su existencia son necesarias tanto las funciones biológicas, como el lenguaje en su uso correcto en el marco de las experiencias sociales (el 
de nivel superior que se requieren en los procesos cognitivos que intervienen en el pensamiento crítico no son posibles si no hay un lenguaje presente. La biología per se no es suficiente para sustentar la ontología de la mente humana. El lenguaje y las experiencias sociales son fundamentales para que cada ser humano tenga conciencia, intencionalidad y creencias en niveles superiores, tal y como lo exige el pensar crítico. Ese uso del lenguaje reglado, intencional y consciente que permite la relación mente-mundo a través de la acción es lo que se ha denominado biopragmatismo.

En este sentido, puede explicarse que el lenguaje está presente en los procesos del entendimiento (racionalidad, juicio, intelecto) y en los estados de la mente humana, puesto que permite que estos evolucionen. En cuanto a la racionalidad es preciso decir que esta depende del uso del lenguaje. Cuanto mayormente complejo sea el lenguaje proposicional y más perfecto su uso, mejores serán los procesos racionales elaborados por los seres humanos. No se puede hablar de razón sin lenguaje, puesto que esta se evidencia en el uso del mismo, lo que no implica decir que la razón se reduce a este. Una persona que arguya y cuestione de mejor manera elabora argumentos más sólidos a la hora de dar solución a los problemas de su entorno y alcanza mejores comprensiones de los planteamientos tanto propios como de otros. Ello se da porque el uso del lenguaje regulado le lleva a analizar las proposiciones y actos de habla usados desde nociones lógicas, con el fin de que su comunicación sea exitosa. Así el uso del lenguaje y los procesos racionales están en íntima relación, puesto que el uso del lenguaje permite alcanzar niveles mayores de racionalidad. Por otra parte, el lenguaje es un elemento que permite expresar y evidenciar en otros seres humanos tales niveles de racionalidad y buen juicio, cosa que las acciones por sí mismas no siempre logran.

Ahora bien, antes de explicar esos tres elementos esenciales del pensamiento crítico, se considera relevante abordar de manera sencilla uno a uno con el fin de comprender mejor, porque se plantean como parte de la naturaleza del pensamiento crítico.

\subsection{Racionalidad, buen juicio e intelecto}

Cada una de las definiciones sobre pensamiento crítico hacen referencia a uno u otro de estos términos en calidad de facultades del pensamiento o del entendimiento, según la postura filosófica que se asuma.

La racionalidad humana está presente en el pensador crítico y se evidencia en sus argumentos, en la resolución que da a los problemas que identifica, en las

\footnotetext{
uso correcto del lenguaje -pragmatismo- vincula el comportamiento intencional de los enunciados, su semántica y por supuesto toda una conducta verbal dominada por reglas). Esta definición es propia. Como neologismo surge de las construcciones que han realizado pensadores analíticos como Searle, Peirce, Wittgenstein, entre otros (autores que brindaron el soporte a este nuevo término, a partir de sus investigaciones en el tema de la mente, el lenguaje y los estudios de la sociedad).
} 
comprensiones del entorno y en la toma de decisiones. La racionalidad lleva a comprensiones de orden epistémico de los diferentes problemas a los que se enfrenta cualquier ser humano: "Pensar críticamente la realidad implica necesariamente acceder a soluciones epistemológicas por sistemas alternos de pensamiento, es decir, por caminos de enriquecimiento cognitivo destinados a repensar las estructuras de toda racionalidad mecánica” (Martín y Barrientos, 2009, 22).

En otras palabras, aprender a pensar críticamente implica aprender a razonar de mejora manera y usar herramientas lógicas al expresar verbalmente los argumentos que nos mueven a tomar decisiones y actuar. Tal vez por ello,

la línea argumentativa del pensamiento crítico propone un tipo de educación basada en competencias argumentativas, propositivas e interpretativas. De este modo, trata de promover un tipo de práctica educativa en la que los alumnos aprendan a saber justificar, apoyar, desarrollar una idea, saber valorar y considerar diversas alternativas, convencer de algo a alguien y, de manera particular también, saber contraargumentar (Martín y Barrientos, 2009, 24).

El razonamiento está directamente relacionado con el conocimiento, con los juicios, con la valoración y se hace efectivo en la expresión verbal en términos de argumentos lógicos. "El pensamiento crítico proporciona a los estudiantes las herramientas necesarias para saber el tipo de conocimiento que deben utilizar en determinada situación, además analiza la estructura y consistencia de los razonamientos de las opiniones o afirmaciones que la gente acepta como verdaderas en el contexto de la vida cotidiana» (Beltrán y Torres, 2009, 68).

Por ello, cuando se habla de que elementos como la razón, el intelecto y el buen juicio están presentes en el pensamiento crítico se entienden estos como facultades que permiten a los seres humanos ser capaces de pensar por sí mismos, a través de un uso correcto y lógico del lenguaje, procesos de pensamiento cuyo contenido epistémico orienta las acciones de forma ética.

El pensador crítico ideal es una persona habitualmente inquisitiva; bien informada,
que confía en la razón; de mente abierta; flexible; justa cuando se trata de eva-
luar; honesta cuando confronta sus sesgos personales; prudente al emitir juicios;
dispuesta a reconsiderar y si es necesario a retractarse; clara respecto a los pro-
blemas o las situaciones que requieren la emisión de un juicio; ordenada cuando
se enfrenta a situaciones complejas; diligente en la búsqueda de información
relevante; razonable en la selección de criterios; enfocada en preguntar, indagar,
investigar; persistente en la búsqueda de resultados tan precisos como las circuns-
tancias y el problema o la situación lo permitan (Facione, 2007, 21).

Ahora bien, si se revisan teorías -racionalistas, incluido el criticismo kantianoen torno a estos tres elementos propios del entendimiento humano se puede evidenciar que tanto la razón como el buen juicio y el intelecto se asumen desde una perspectiva naturalista, es decir, tales facultades son propias a la naturaleza humana; una perspectiva que se sustenta biológicamente, la cual, en la actualidad 
es defendida y sustentada desde los estudios neurofisiológicos que aportan las ciencias cognitivas. Pese a ello, este estudio pretende plantear al lenguaje como condición necesaria para la existencia de la racionalidad y del buen juicio, así como parte esencial de las virtudes intelectuales que plantea Facione, para lo cual se propone una concepción biopragmática para la racionalidad.

Así, se plantea una relación lenguaje-razón expuesta desde algunos planteamientos de Wittgenstein y Searle, quienes contrario a Kant, no apuestan por la razón imperativa que regula el uso del lenguaje, sino por una racionalidad alcanzada en el uso del lenguaje. El uso reglado y lógico de lenguaje es el que permite evidenciar la razón. Para estos autores analíticos, en primera instancia, desde la misma naturaleza humana el hombre está dotado para crear sonidos, para crear palabras y para crear nombres, y con el uso del lenguaje se requiere entonces del desarrollo de la razón. Desde esta postura, primero está en el ser humano el lenguaje que la misma razón, ya que, si el lenguaje es pensamiento y este posee sentido en su uso, la razón se va desarrollando a la par que se desarrolla el pensamiento con el uso del lenguaje. Tal vez pueda decirse que en Wittgenstein la razón también sea el lenguaje mismo, pues no habría procesos racionales si no hay uso del lenguaje. Y el uso se da en el ámbito social en las experiencias, en la pragmática, mientras que, para Searle, la racionalidad es una cualidad derivada de la intencionalidad.

En este sentido, no es la razón, tal y como lo ha expuesto la tradición filosófica, la que descubre las propiedades internas de la realidad, sino que es el ser humano en el análisis de la proposición el que realiza tal trabajo. Se podría decir que la relación proposición-realidad está mediada por el uso de la lógica (de la razón como principio lógico del lenguaje mismo [no como principio imperativo]), es decir, que el lenguaje exige de un uso correcto no solo en la comunicación ordinaria, sino en la comunicación de la realidad en nuestro pensamiento; por lo cual, al hacer tal uso se requiere de un análisis permanente de las proposiciones usadas, y a esto podríamos llamarle razón (al uso correcto del lenguaje en el develamiento de las proposiciones que usamos para referirnos al mundo). El mundo no puede ser pensado sin lenguaje y es el lenguaje el que figura al mundo. Nuestro conocimiento del mundo, según Wittgenstein, está demarcado por el lenguaje que usamos para referirnos al mismo. Así, la función de la proposición acaba por comunicar el estado de las cosas y la racionalidad nos permite entender el lenguaje que usamos, usar la lógica en el mismo lenguaje con el fin de desvelar los errores en el conocimiento (errores propiciados por el mal uso del lenguaje). En otras palabras, la razón no es la facultad máxima de la condición humana, sino la cualidad máxima del pensamiento (entendiendo el pensamiento como lenguaje). Tal vez por ello, Wittgenstein, alejándose un poco de Kant, expone: «Los límites de mi lenguaje significan los límites de mi mundo" (Wittgenstein, 2009, af: 5.6), con el fin de mostrar que la razón por sí sola no traza los límites en el conocimiento, sino que se hace en la relación lenguaje-racionalidad. 
En esta misma línea de trabajo, podemos encontrar los aportes de la filosofía analítica de Searle. En este filósofo se evidencia un cambio fuerte en la concepción tradicional de razón. Este pensador no concibe la razón como facultad humana, sino como una cualidad de la Intencionalidad, la cual se desarrolla gracias al lenguaje. La teoría de los actos de habla plantea una nueva relación entre el lenguaje y la mente, pero ante todo inicia un nuevo planteamiento frente a la relación lenguaje-razón. Planteamiento que Searle desarrollará más adelante en Rationality in Action (2001). Para Searle la pregunta acerca de la posibilidad de concebir el pensamiento sin lenguaje o el lenguaje como creador del pensamiento debe ser solucionada en la relación existente entre las expresiones que usamos y nuestros estados mentales. Los estudios iniciados por Searle, en Speech Acts, se enmarcan en la línea del análisis de las dimensiones del acto lingüístico, iniciados por Austin (1962), se enmarcan en el hablante y con ello en sus estados mentales, específicamente en la intencionalidad que cada acto desvela. El análisis del lenguaje, en este nuevo planteamiento, se realiza en la relación lenguaje-intencionalidad, más que en la relación lenguaje-razón, pero que permite afirmar el planteamiento de que la razón es de naturaleza biopragmática, al menos la razón que se espera alcance el pensador crítico, para que sea un agente capaz de analizar, interpretar, inferir, explicar y evaluar, tal y como lo expone Facione (2007) siguiendo la taxonomía de Bloom.

\subsection{Conciencia, intencionalidad y creencias}

Siguiendo los planteamientos de Facione (2007) podríamos decir que elementos como la autorregulación y la evaluación de toma de decisiones, situaciones, contexto y argumentos son esenciales al pensamiento crítico. Por ello, se hace indispensable hacer su análisis, el mismo que nos direcciona hacia los estados de la mente: Conciencia, intencionalidad y creencias. Son tales estados mentales los que hacen posible que un sujeto se autorregule y evalúe o valore ${ }^{15}$. En este apartado es preciso decir que, si bien estos elementos son objetos de estudios propios de la filosofía del lenguaje, aparecen en los procesos de pensamiento crítico como componentes esenciales del mismo.

Podríamos poner la evaluación o valoración en el apartado anterior, es decir, con la racionalidad, el buen juicio y el intelecto -y estaríamos en lo correcto-;

Ilustración significa el abandono por parte del hombre de una minoría de edad cuyo responsable es él mismo. Esta minoría de edad significa la incapacidad para servirse de su entendimiento sin verse guiado por algún otro. Uno mismo es el

15. Se analizan los estados mentales aparte de la racionalidad no porque efectivamente así se presenten los procesos de pensamiento en el ser humano, sino porque para este estudio es importante demarcar el papel de unos y otros en el pensar crítico. De otra parte, es importante aclarar que el pensamiento humano en su complejidad relaciona la razón con la mente, aun cuando no son lo mismo. 
culpable de dicha minoría de edad cuando su causa no reside en la falta de entendimiento, sino en la falta de resolución y valor para servirse del suyo propio sin la guía del de algún otro. Sapere aude! (Kant, 2013, Ak. VIII, 35).

No obstante, frente a la condición expuesta por Kant, Hegel plantea que "cuando se está clavado en el sistema de la opinión y del prejuicio, la única diferencia entre estarlo por la autoridad de otros o por convicción propia es la vanidad de esta última» (Hegel, 2010 [PdG], 56). Con lo que se opone a la racionalidad trascendental como única condición para la crítica.

Lo anterior nos lleva a considerar que, pese a que es evidente que se espera que el pensador crítico evalúe racionalmente, es preciso decir que la conciencia, como estado mental de nivel superior, es mayormente influyente en el evaluar que la misma razón. Un pensador crítico debe ser consciente de sus valoraciones, juicios y evaluaciones, de lo contrario, no podríamos decir que sus decisiones y juicios valorativos son críticos. Ello atiende a que cuando hacemos referencia a la evaluación es preciso entenderla como la «valoración de la credibilidad de los enunciados o de otras representaciones que recuentan o describen la percepción, experiencia, situación, juicio, creencia u opinión de una persona; y la valoración de la fortaleza lógica de las relaciones de inferencia, reales o supuestas, entre enunciados, descripciones, preguntas u otras formas de representación» (Facione, 2007, 5). Lo que involucra al sujeto no solo en la racionalidad, sino en sus experiencias, en sus percepciones y creencias, las cuales deben ser conscientes.

La evaluación crítica involucra estos tres estados mentales. Puesto que al evaluar todo sujeto lo hace de acuerdo con sus conocimientos, los mismos que se presentan en forma de creencias expresadas en términos lingüísticos. Por otra parte, la evaluación no es algo que se haga arbitrariamente, un pensador crítico evalúa de forma intencional, puesto que dirige su atención sobre el objeto -la situación o el argumento expuesto-. La intencionalidad del sujeto al evaluar el entorno y las condiciones de posibilidad del conocimiento del mismo se evidencia en los procesos y habilidades anteriormente descritas, puesto que, al analizar, interpretar, inferir y explicar algo, cada sujeto lo expresa en su lenguaje a través de actos de habla ilocucionarios, expresados en los argumentos que arguye.

Ahora bien, de la misma manera que el sujeto crítico usa sus estados mentales para evaluar, lo hace al momento de autorregularse. Para Facione (2007)

los expertos definen el significado de la autorregulación como monitoreo autoconsciente de las actividades cognitivas propias, de los elementos utilizados en esas actividades, y de los resultados obtenidos, aplicando particularmente habilidades de análisis y de evaluación a los juicios inferenciales propios, con la idea de cuestionar, confirmar, validar, o corregir el razonamiento o los resultados propios. Las dos subhabilidades, en este caso, son el autoexamen y la autocorrección (p. 6).

Una definición que involucran a la conciencia, la intencionalidad y las creencias de manera directa, pues el ser humano es capaz de regular sus comportamientos y pensamientos en la medida en que lo hace consciente e intencionalmente. 
El monitoreo de autorregulación que sigue Facione (2017) permite al sujeto monitorear sus procesos cognitivos y, siguiendo a Paul (2008), pasar de la creencia a la acción. En este sentido, las creencias y los razonamientos que estas suscitan se convierten en motivos para la acción, de tal forma que, al pensar críticamente, priman los procesos mentales y racionales sobre los deseos, es por ello que se alcanzan planteamientos razonados y lógicos a los problemas que acontecen en el diario vivir. La autorregulación conlleva que cada sujeto sea responsable y autónomo, tal y como lo propone Kant.

Desde esta concepción que relaciona racionalidad y mente se teje un modelo crítico que sustenta las razones para actuar, un modelo que, siguiendo a Searle (2001) es contrario al modelo clásico del deseo. Las razones son creadas racional, intencional y consciente e independientemente del deseo. En las acciones realizadas por un pensador crítico -más que el componente sicológico (deseos) por el que se orienta la teoría tradicional de la razón- existen componentes como el conocimiento expresado en las creencias, que puede llegar a ser una razón para actuar.

Desde esta propuesta del giro lingüístico sustentada en filósofos de la mente como Russell, Wittgenstein, y ante todo fundamentada en Searle, se propone una concepción de racionalidad crítica diferente a la expuesta por Kant. Según Searle, en Rationality in Action, no puede hacerse un estudio de la razón separada de la mente y sus estados, y de los aportes de las ciencias cognitivas; tampoco puede hacerse un estudio de la mente y del pensamiento separado del lenguaje. Si en la formación de un pensador crítico, como proponen Facione (2007) y Paul (2008), la razón se evidencia en la acción, entonces hay que indagar por los procesos mentales que se requieren para lograr con éxito la acción misma, y al indagar se identifican pensamiento, lenguaje y acción en una interrelación constante. Ya que, el lenguaje, como lo expuso Kenny (1989), es el elemento que permite reconocer las razones que posee el agente para actuar. Quien no posea lenguaje no puede explicar sus razones, por lo cual, sería imposible desvelar la relación pensamientoacción. En este sentido, a un animal que no posea lenguaje no puede atribuírsele intencionalidad en sus acciones, ni conciencia en niveles superiores, así como tampoco razonamientos y argumentos, es decir, no puede atribuírsele pensamiento crítico.

\section{Ontología BIOPRAGMÁtica DEL PENSAMIENTO CRÍTICO: LENGUAJE, PENSAMIENTO Y ACCIÓN}

Tal y como se ha expuesto hasta el momento, hay tres elementos fundamentales en la naturaleza del pensamiento crítico, aun cuando en muchos de los discursos tal vez se abogue por dos de ellos (pensamiento y lenguaje) más que por la acción misma; sin embargo, como lo expone Paul (2008), la acción es un elemento fundamental en el cual el pensador crítico evidencia los niveles superiores alcanzados que lo han llevado a asumir posturas argumentadas, lógicamente válidas y que orientan su comportamiento. 
Es preciso decir, entonces, que una vez trazada la relación directa entre facultades del pensamiento y estados mentales, sustentada por Searle (2001, 2010 y 2015), se puede entender mejor que los pensadores críticos no solo usan la razón ilustrada, tal y como lo expuso Kant, para alcanzar la autonomía, sino que además son conscientes, argumentan y actúan intencionalmente y el conocimiento o sus creencias verdaderas se constituyen en razones para actuar.

Ahora bien, en esa relación lenguaje, pensamiento y acción podemos ubicar el pensamiento crítico en el ser humano y no concebirlo como entidad autónoma, como en algunos discursos se hace; de igual forma, se concibe al ser humano anclado a un contexto, a un devenir histórico, a una realidad que necesita conocer y comprender para vivir de mejor manera y habitar en el mundo con una postura autónoma. Por ello, identificar que el pensamiento crítico como fenómeno tiene una ontología biopragmática, puesto que deviene tanto de la biología (bio), pues el pensamiento (racionalidad, buen juicio, intelecto, conciencia, intencionalidad, creencias y lenguaje) en parte emerge de procesos neurofisiológicos, así como también se desarrolla a través de procesos sociales, en el uso regulado e institucionalizado del lenguaje, en las experiencias con los otros, con el mundo, es decir, que también es pragmático ${ }^{16}$, puesto que lleva a la acción, nos permite pensar en las formas o estrategias que se deben usar para alcanzar desarrollo de habilidades de nivel superior, en la que los sujetos se comprometen con sus creencias, razonamientos, uso del lenguaje y acciones de manera consciente e intencional. En otras palabras, sus decisiones, razones y acciones están en coherencia, son racionales y razonables.

Asimismo, identificar la naturaleza biopragmática del pensamiento crítico nos permite, además de comprender la relación lenguaje, pensamiento y acción que se da en el sujeto, comprender que pensar y actuar de manera crítica lleva en su transfondo lo que lo que Searle denominó dirección de ajuste, en dos sentidos mente-mundo y mundo mente (Searle, 1969) y que como docentes debemos propiciar espacios en los cuales se logre de manera exitosa esa dirección de ajuste, para mejores comprensiones y soluciones a los problemas que el entorno nos plantea o que en ocasiones creamos por pseudoconocimientos que consideramos verdaderos.

Es substancial aclarar que, en los procesos del pensamiento que llevan al pensar y actuar crítico, las creencias tienen de fondo una dirección de ajuste mente-mundo, que fundamenta las razones para argumentar, plantear soluciones

16. Es preciso aclarar que esta postura no se asume desde la concepción del pragmatismo utilitarista, sino desde el pragmaticismo propuesto por Peirce, en el cual el uso correcto del lenguaje es esencial para la acción. El mismo que retoman el Wittgenstein de las Investigaciones filosóficas y Searle en su teoría de Actos de habla y en La construcción de la realidad social. De igual forma, es preciso aclarar que parte del emergentismo biológico que se asume en esta propuesta es desde los planteamientos searleanos, en su teoría de El redescubrimiento de la mente (1992), más que desde sus planteamientos iniciales en Intentionality (1983). Se afirma que gran parte, más no todo, porque para Searle, la ontología de la realidad social, del pensamiento y de la mente se sustenta en la biología, mientras que en una ontología biopragmática se apuesta por elementos tanto biológicos como sociales, puesto que el mismo lenguaje es un elemento biosocial. 
razonadas y razonables y actuar; mientras que en las intenciones (que están directamente relacionadas con la acción) la relación se presenta mundo-mente. En ambos casos, el lenguaje es el que permite que tal relación sea efectiva. Así "las nociones de condiciones de satisfacción y dirección de ajuste se aplican a las dos entidades mentales y lingüísticas» (Searle, 2001, 52). Por lo cual, existe un paralelismo entre la naturaleza de la mente y la naturaleza de los actos lingüísticos, mediada por la racionalidad. De acuerdo con lo anterior, la dirección de ajuste además de ser esencial para comprender la relación entre la intencionalidad y el mundo es esencial para comprender las acciones que se efectúan en el mundo; es decir, para desvelar los procesos racionales y mentales que realizan los sujetos críticos, los cuales se evidencian en las acciones.

Lo anterior nos muestra que ser un pensador crítico no solo es razonar de manera lógica, sino actuar de acuerdo con lo que Facione expone como el buen juicio. En tal sentido, todo docente debe comprender que los razonamientos y argumentos expresados por sus estudiantes, también deben reflejarse en las acciones, en las tomas de decisiones, en la convivencia y en la forma de actuar con los otros seres humanos en el mundo; por ello, cuando un pensador crítico realiza una acción $\mathrm{x}$ inicia un proceso mental que involucra razón, intencionalidad, conciencia y creencias, procesos mentales que le exige deliberar, y en cada uno de esos procesos de nivel superior intervienen elementos como: lenguaje, pensamiento y una serie de conocimientos de los cuales el agente es consciente para lograr la acción.

La naturaleza biopragmática del pensamiento crítico permite entender que tener intenciones conscientes y autorreguladas exige al ser humano un proceso racional, en el cual se involucra la reflexión y la toma de decisión para tener tal intención y con ello, alcanzar las mejores soluciones argumentadas para los problemas que se le presentan en su vida cotidiana, con el fin de realizar las acciones más convenientes tanto para él como para los demás con quienes comparte. En otras palabras, tener la creencia de $p$; así como tener deseos sobre $p$, demanda al pensador crítico iniciarse en una serie de procesos mentales de orden superior y razonamientos sobre la creencia y el deseo que posee, con el fin de pasar de manera sustentada a tener la intención de $p$ y si su intención de $p$ cumple con las condiciones de satisfacción dadas por la dirección de ajuste mundo-a-mente, efectivamente realizará la acción $p$, de manera autorregulada y monitoreada. Realizar la acción de participar activamente en la democracia, por ejemplo, y dar soluciones argumentadas como ciudadano exige al sujeto crítico tener la creencia sobre lo que es la democracia, además de tener la intención consciente de su participación democrática. Si la intención de participar y la creencia de lo que es participar de una democracia cumplen con ciertas condiciones de satisfacción en las cuales el mundo se ajusta a la intención y a las razones que tiene cada sujeto para ser un agente político que participa de manera activa en la democracia, entonces se llevará a cabo tal acción. El vínculo causal, la intención-en-la-acción, es la que hace efectivo el movimiento corporal del ejercicio de la democracia. 


\section{PENSAMIENTO CRÍTICO COMO CONSTRUCCIÓN COLECTIVA}

Una vez desvelada la ontología biopragmática del pensamiento crítico y comprender que en su naturaleza pensamiento, lenguaje y acción son condiciones necesarias para su existencia, además de ser elementos que devienen tanto de la biología como de lo social, se puede comprender que ser pensador crítico no es algo innato, sino algo que se logra en el diálogo con otros y con el mundo. El pensamiento crítico se alcanza en las construcciones colectivas. Un sujeto per se sin usar adecuadamente el lenguaje y la lógica, sin actuar en un entorno social, sin discutir y argumentar con otros y sin justificar sus acciones no logra pensar críticamente.

La crítica se logra en la discusión, en el planteamiento de argumentos, en el cuestionamiento sobre lo que otros han expuesto; de lo contrario, no tendría sentido una crítica de "yo" consigo mismo, si no hay otros puntos de referencia y otras formas de pensar diferentes a las propias. ¿Quién discutiría, evaluaría y juzgaría un discurso, una acción y un argumento si no hay interlocutores ni criterios consensuados y normativos para ello? Por ello, cuando se inician procesos formativos en pro del pensamiento crítico es un requerimiento fundamental crear métodos que favorezcan, además de la autorregulación, la discusión y la reflexión.

No podemos esperar un pensamiento crítico enmarcado en un sujeto desde una concepción solipsista. Para desarrollar habilidades que tienden a la crítica es indispensable que haya perspectivas alternas sobre las cuales discutir, otros abordajes conceptuales que difieran con los propios, otras miradas con otros criterios. Si el pensamiento crítico solo se realizase con el ejercicio de la razón individual, entonces ¿con una serie de ejercicios de entrenamiento lógico y aprender a argumentar por escrito siguiendo planteamientos lógicos y un amplio conocimiento sobre un tema, sería suficiente? Me atrevo a afirmar que no. El desarrollo de habilidades de nivel superior para pensar críticamente se genera en medio de la reflexión, del diálogo, de la discusión con el otro, con los textos y contextos, con las ideas contrarias que permitan iniciar contraargumentos y nuevos planteamientos. Formar un criterio propio y alcanzar la madurez y la autonomía intelectual para pensar críticamente demanda un ejercicio dialógico, un correcto uso del lenguaje y una buena argumentación que solo se alcanza en la interacción social, en la construcción colectiva.

La naturaleza biopragmática del pensamiento crítico ostenta el elemento social como condición de posibilidad del mismo. Cuando se plantea que la mayéutica y la dialéctica, entre otros métodos, propician espacios para jalonar habilidades de pensamiento de orden superior, se intuye que tales métodos abren el espacio para reflexionar, discutir y argumentar con el otro u otros. Es cierto que en los procesos cognitivos de cada sujeto se genera el jalonamiento de las habilidades que se requieren para pensar críticamente, pero ello no es posible si el sujeto está solo, aislado, sin procesos comunicativos con otros seres humanos, con otros textos, que le posibiliten jalonar desarrollos en la argumentación y la lógica. No tiene sentido hablar de un sujeto que desarrolla pensamiento crítico si no usa correctamente el 
lenguaje para debatir y discutir con otros. Si sustentamos el pensamiento crítico en el marco meramente biológico de un sujeto, sin contar con el elemento social, es decir, un sujeto que tiene arraigadas sus creencias, ideas y perspectivas sobre un tema y que no entra en interacción y discusión con otras perspectivas y creencias alternas, estaríamos desconociendo la naturaleza social que subyace al pensar de forma crítica, pues ¿a quién le discute, para qué argumenta, para qué dar solución a problemas? En una perspectiva solipsista el pensar críticamente no tiene sentido.

El lenguaje y la mente como elementos biosociales se convierten en condiciones necesarias para la existencia del pensamiento crítico. Sin una pragmática correcta del lenguaje, sin discusión, sin reflexión y debate, no es posible considerar que alguien llegue a pensar críticamente, pues sus estados mentales no alcanzarán los desarrollos de nivel superior que se requieren y las habilidades pretendidas.

\section{CONClusión}

En la base natural del pensamiento crítico podemos encontrar tres elementos que constituyen su ontología: lenguaje, pensamiento y acción. Elementos que permiten el desarrollo de habilidades de nivel superior para razonar, tomar decisiones y actuar de manera racional y razonable. El lenguaje como elemento central y eje relacional entre el pensamiento y la acción es el que hace posible alcanzar grados superiores de racionalidad, intencionalidad y conciencia y, con ello, permite alcanzar ciertas estructuras del pensamiento para pensar y actuar críticamente.

Lenguaje, pensamiento y acción son elementos biosociales que en el pensador crítico -en la relación mente-mundo- se evidencian en la coherencia entre lo que piensa, dice (argumenta) y hace intencionalmente. En otras palabras, la ontología del pensamiento crítico es biopragmática, puesto que vincula tanto elementos propios de la biología humana, como elementos sociales que se evidencian en la forma de pensar, decir y actuar.

El reconocimiento de una ontología biopragmática del pensamiento crítico exige al maestro dejar de pensar en procesos formativos con base en el seguimiento de reglas lógicas, bajo el uso de lenguajes solipsistas, y dar el giro hacia una formación en espacios sociales, con el fin de generar discusiones en espacios contextuales y problemáticos en los que cada sujeto desarrolle las herramientas mentales necesarias para dar soluciones argumentadas a los problemas evidenciados; soluciones que se alcanzan en la discusión con el otro al llegar a consensos que transformen la realidad.

De acuerdo con lo anterior, una educación que forme a los sujetos para pensar por sí mismos de forma crítica exige a los maestros conocer la ontología biopragmática del pensamiento crítico, con el fin de planear intencionalmente estrategias didácticas que propendan por el desarrollo de las habilidades necesarias para que los sujetos sean agentes activos en discusiones y reflexiones que los enfrenten a la realidad social que les aqueja. Visto así, formar pensadores críticos es formar 
ciudadanos conscientes y capaces de tomar decisiones propias, justificadas y responsables que conlleven acciones y les permitan vivir de mejor manera.

\section{REFERENCIAS BIBLIOGRÁFICAS}

Beltrán, M. J. y TORReS, N. Y. (2009) Caracterización de habilidades de pensamiento crítico en estudiantes de educación media a través del test HCTAES. Revista del Instituto de Estudios en Educación de la Universidad del Norte, 11 (1), 65-85. Recuperado de: http:// rcientificas.uninorte.edu.co/index.php/zona/article/viewFile/1595/1045.

Bloom, B.; Furst, E.; Hill, W. y Krathwohl, D. R. (1956) Taxonomy of Educational Objectives: Handbook 1. The cognitive Domain. Addison-Wesley.

Descartes, R. (2014) Meditaciones acerca de la Filosofía Primera. Trad. 1. a y 2. ${ }^{a}$ ed. Jorge Aurelio Díaz. Edición trilingüe. Bogotá, Colombia, Universidad Nacional de Colombia.

FaCione, P. (1990) Critical Thinking: A Statement of Expert Consensus for Purposes of Educational Assessement and Instruction. Proyecto Delphi. Recuperado de: http://files.eric. ed.gov/fulltext/ED315423.pdf. American Philosophical Association Asociación Norteamericana de Filosofía.

Facione, P. (2007) Pensamiento crítico: ¿qué es y por qué es importante? Trad. María Cecilia Bernat. Eduteka. Recuperado de: http://eduteka.icesi.edu.co/pdfdir/PensamientoCriticoFacione.pdf.

France, D. et al. (2003) El lugar del relativismo en los intercambios filosóficos de los alumnos. Perfiles Educativos, 24 (96), 5-32. Recuperado de: http://www.redalyc.org/articulo. oa?id=13209602\#.

Hegel, G. (2010) $[P d G]$. Fenomenología del espíritu. Trad. Antonio Gómez Ramos. Edición bilingüe. Madrid, España, Abada.

Jaeger, W. (1980) Paideia: los ideales de la cultura griega. Trad. Joaquín Xirau y Wenceslao Roces. México D. F., México, Fondo de la Cultura Económica.

Kant, I. (2011) [krV]. Crítica de la razón pura. Trad. Mario Caimi. Edición bilingüe. México, D. F., Fondo de Cultura Económica, Biblioteca Immanuel Kant.

Kant, I. (2013) ¿Qué es la ilustración? Trad. Roberto R. Aramayo. Madrid, Alianza.

Kenny, A. (2000) La metafísica de la mente. Trad. Francisco Rodríguez Consuegra. Barcelona, Paidós.

López, G. (2012) El pensamiento crítico en el aula. Docencia e Investigación, 22 (1), 41-60. Recuperado de: http://www.ipesad.edu.mx/repositorio1/BG-B04-19.pdf.pdf.

Martín, A. y Barrientos, O. (2009) Los dominios del pensamiento crítico: una lectura desde la teoría de la educación. Teoría de la Educación, 21 (2), 19-44. Recuperado de: http:// revistas.usal.es/index.php/1130-3743/article/download/7150/7215.

MarX, K. (2014) La crítica como fundamento, en Textos selectos: Textos de filosofía, política y economía. Manuscritos de París. Manifiesto del Partido Comunista. Crítica del programa de Gotha. Biblioteca de Grandes Pensadores. Madrid, España, Editorial Gredos, S. A., 75-151.

Ministerio DE EdUCACIÓn NACIONAL (2009) Competencias genéricas en educación superior. Educación Superior [boletín informativo], n. ${ }^{\circ}$ 13. Recuperado de: http://www.mineducacion.gov.co/1621/articles-92779_archivo_pdf_Boletin13.pdf. 
Montoya, J. (2007) Acercamiento al desarrollo del pensamiento crítico, un reto para la educación actual. Revista Virtual Universidad Católica del Norte, 21 (1), 317-333. Recuperado de: http://revistavirtual.ucn.edu.co/index.php/RevistaUCN/article/viewFile/165/317.

NiCKERSON, R. (1994) The teaching of thinking and problema solving, en STERNBERG R. J. (ed.) Thinking and problem solving. San Diego, CA, Academic Press, 409-449.

Olivares, S.; SaIz, C. y Rivas, S. F. (2013) Encouragement for Thinking Critically. Electronic Journal of Research in Educational Psychology, 11 (2), 367-394. Recuperado de: https:// www.researchgate.net/profile/Sonia_Olivares_Moral/publication/269602858_Encouragement_For_Thinking_Critically/links/56bb01ef08ae2567351eeba1/EncouragementFor-Thinking-Critically.pdf.

PAUl, R. y Elder R. (2003) Una mini-guía para el pensamiento crítico, conceptos y berramientas. Fundación para el Pensamiento Crítico. Recuperado de: http://www.criticalthinking.org/resources/PDF/SP-ConceptsandTools.pdf.

PORTO, A. (2000) Diseño curricular. Desarrollo y preparación de programas para el estudiante muy capacitado", en Benito, Y. (ed.) Problemática del niño superdotado. Salamanca, Amarú, 85-110.

RodríGuez, A. (2016) Hacia una visión biopragmatista de la conciencia. Eikasía, 68, 207-234. Recuperado de: http://www.revistadefilosofia.org/68-08.pdf.

SeArle, J. (1969) Speech Acts. An essay in the philosophy of language. Cambridge, Cambridge at the University Press.

SEARle, J. (2001) Rationality in Action. Cambridge, MIT Press.

SeArle, J. (2010) Making the Social World. The Structure of Human Civilization. New York, Oxford University Press.

SEARLE, J. (2015) Seeing thinks as they are: a theory of perception. Oxford, Oxford University Press.

Shannon, H. y Allen, T. (2001) Eficacia del programa de instrucción ReBT en el aumento de rendimiento en estudiantes de matemáticas de secundaria. RET, Revista de Toxicomanias, 29 (1), 10-17. Recuperado de: http://www.cat-barcelona.com/uploads/rets/ RET29_2.pdf.

Tamayo, O.; Vasco, C.; Suárez, M.; Quiceno, H.; García, L. y Giraldo, A. (2011) La clase multimodal y la formación y evolución de conceptos científicos a través del uso de tecnologías de la información y la comunicación. Manizales, Universidad Autónoma de Manizales.

TAMayo, O.; Zona, J. y LoAiza, Y. (2014) El pensamiento crítico en el aula de ciencias. Manizales, Editorial de la Universidad de Caldas.

UnESCO (1996) Treasure Within [Report to Unesco of International Commission on Education for the Twenty-first Century]. Paris, Unesco Publishing. Avaible to: http://www.unesco. org/education/pdf/15_62.pdf.

Wacquant, L. (2006) Pensamiento crítico y disolución de la doxa: entrevista con Loïc Wacquant. Antípoda 2, 43-50. Recuperado de: http://www.redalyc.org/articulo. oa? id $=81400203$.

Wittgenstein, L. (2009) Tractatus logico-philosophicus, en Reguera, I. (ed.) Obra completa. Vol. 1. Tractatus logico-philosophicus. Investigaciones filosóficas. Sobre la certeza. Edición bilingüe. Biblioteca de Grandes Pensadores. Madrid, España, Editorial Gredos.

Zoller, U. (1991) La solución de problemas de U y la paradoja de solución de problemas. Asuntos conceptuales en la educación ambiental. Nueva York, Peter Lang. 